\title{
Rene K. Marti, Ronald J. van Heerwaarden (eds): Osteotomies for post-traumatic deformities
}

\author{
Thieme Verlag, New York, Stuttgart, 2008, 704 pp, number. figures, \\ Hardcover, Euro (D) 6-299.95 CHF 498.00, ISBN: 978-3-13-148671-4
}

\author{
Pierre Kehr
}

Received: 28 February 2010 / Accepted: 1 March 2010 / Published online: 16 March 2010

(C) Springer-Verlag 2010

It is about one beautiful hardcovered book of 700 pages; it is entirely devoted to the osteotomies for after-effects of traumatisms and Co-writing by 40 primarily Dutch, Swiss and German surgeons; there are also some American, Japanese, Argentinian and Brazilian, all the authors are under the direction of R. K. Marti and R. J. van Heerwaarden, which are the old and current "popes" of the no-prosthetic surgery of rebuilding in the Anglo-Saxon world. The work was published by the foundation of the AO what one feels so much by the plan of the work than the rigor of work and by the choice of the implants. The book is divided into two parts with initially part of general principles on 55 pages then a review of the various possible anatomical localizations through the skeleton.

The first part, written by the editors in chief to which C. van den Werken, current president of AO Foundation is joined, makes a full overview of what it is necessary to know about the vicious cal of the various bones, the way of studying them on the geometrical plan and the radiological correspondence; the authors then endeavor to define a surgical strategy always based on a planning, a preoperative selection of the implants and a technical tactic good prepared leaving only little place to the per-operational improvisation, as that must be in the majority of the cases.
The second part is a sum of all that a surgeon can meet in his career, with precise clinical cases, documented well, for each anatomical localization. The level of different the chapter, individually, can be only a little unequal considering the number of different authors but is overall very good. It is necessary to make use of it like one thesaurus with the request; it can render service as well to the surgeon still little experienced in particular for the vicious cal most frequently met, as the senior experienced considering the scarcity of certain lesions described in the book. One should not await large-thing of the ways initially, however, because they are only evoked and require to be known in addition. The choice of the implants is varied enough and can be followed without much concern because of its quality and is well adapted in almost all the cases with described pathology.

In short, this book is not read in once beginning with the end, but will quickly become a reference to be consulted by any surgeon who wants to make osteotomies for the often difficult problems arising from the vicious cal of all kinds.

Claude Schwartz Colmar (France)

No funds were received in support of this study.

P. Kehr $(\bowtie)$

SOTEST, Strasbourg, France

e-mail: kehrpier@aol.com 